\title{
Un secret bien gardé : l'inceste dans la société traditionnelle québécoise, 1858-1938
}

\section{Marie-Aimée Cliche}

Volume 50, numéro 2, automne 1996

URI : https://id.erudit.org/iderudit/305508ar

DOI : https://doi.org/10.7202/305508ar

Aller au sommaire du numéro

Éditeur(s)

Institut d'histoire de l'Amérique française

ISSN

0035-2357 (imprimé)

1492-1383 (numérique)

Découvrir la revue

Citer cet article

Cliche, M.-A. (1996). Un secret bien gardé : l’inceste dans la société traditionnelle québécoise, 1858-1938. Revue d'histoire de l'Amérique française, 50(2), 201-226. https://doi.org/10.7202/305508ar
Résumé de l'article

Cette étude sociale de l'inceste a été réalisée à partir d'archives judiciaires. La correspondance du procureur général de la province et les archives des tribunaux de la région de Québec en recèlent 217 cas incluant 131 procès et 95 condamnations. Plus des deux tiers sont des cas d'inceste entre père et fille. L'isolement en milieu rural et la dépendance économique à l'égard du père incitaient bien des familles à garder cette situation secrète, mais les victimes pouvaient recourir à la parenté, aux voisins et au curé. Ces dossiers révèlent une relation de pouvoir brutale entre hommes et femmes au sein de la famille traditionnelle. 


\title{
UN SECRET BIEN GARDÉ L'INCESTE DANS LA SOCIÉTÉ TRADITIONNELLE QUÉBÉCOISE 1858-1938
}

\author{
MARIE-AIMÉE CLICHE \\ Chercheure associée \\ Département d'histoire \\ Université du Québec à Montréal
}

\section{RÉSUMÉ}

Cette étude sociale de l'inceste a été réalisée à partir d'archives judiciaires. La correspondance du procureur général de la province et les archives des tribunaux de la région de Québec en recèlent 217 cas incluant 131 procès et 95 condamnations. Plus des deux tiers sont des cas d'inceste entre père et fille. L'isolement en milieu rural et la dépendance économique à l'égard du père incitaient bien des familles à garder cette situation secrète, mais les victimes pouvaient recourir à la parenté, aux voisins et au curé. Ces dossiers révèlent une relation de pouvoir brutale entre hommes et femmes au sein de la famille traditionnelle.

\begin{abstract}
This article is a social analysis of incest based on judicial archives. Two hundred and seventeen cases, including one hundred and thirty-one trials leading to ninety-five convictions, have been uncovered in the correspondance of the Attorney-General of the province and in the archives of the judicial districts of Québec, Montmagny, la Beauce and La Malbaie. More than two thirds of the cases involve incest between father and daughter. Rural isolation and the economic dependence of the family on the father made many families keep these affairs a secret, although the victims did have the support of their relatives, neighbours, and the parish priest. These court documents reveal the brutal nature of the power relations between men and women at the heart of the traditional family.
\end{abstract}

1. Ce travail a été rendu possible grâce à une subvention du Conseil de recherches en sciences humaines du Canada obtenue par l'intermédiaire de l'Université de Montréal à titre de chercheure invitée. Nous remercions Sarah Filotas et Isabelle-Audrey Tardif qui nous ont secondée dans le dépouillement des archives. Tous nos remerciements aussi aux historiens Serge Gagnon, Jean-Marie Fecteau, Louise Dechêne et Jean-Claude Robert pour leurs conseils et leurs commentaires.

RHAF, vol. 50, n 2, automne 1996 
Parmi les problèmes sociaux qui retiennent actuellement l'attention des spécialistes aussi bien que celle du grand public, celui des abus sexuels faits aux enfants et aux adolescents figure en bonne place. Les travailleurs sociaux et les psychologues qui s'efforcent de remédier à la violence familiale, et le mouvement féministe qui dénonce l'exploitation sexuelle des femmes, ont contribué à sensibiliser la population à ce problème. Aujourd'hui, des centaines de personnes révèlent les abus dont elles ont été victimes dans un passé pas très lointain, soit au sein de leur famille ou dans des établissements religieux. Ces dénonciations prennent la forme de poursuites judiciaires et de récits de vie, parfois d'une grande qualité littéraire 2 .

Jadis considéré comme le secret le mieux gardé des familles, l'inceste est, depuis une vingtaine d'années, étudié sous toutes ses facettes par des chercheurs de différentes disciplines. Au début, certains ont cru y voir un phénomène marginal, plus fréquent à la campagne, relié à la pauvreté, à l'alcoolisme, voire à la déficience mentale ${ }^{3}$. Des études plus fouillées ont rapidement révélé que ce genre d'abus existait autant en milieu urbain et dans toutes les classes sociales ${ }^{4}$. Parmi les différentes façons d'aborder ce problème, signalons l'approche psychanalytique, marquée par l'influence de Freud et sa théorie des pulsions; l'approche de la famille dysfonctionnelle qui considère l'inceste comme une conséquence des problèmes familiaux et non l'inverse, et qui en attribue la responsabilité en grande partie à la mère; l'approche psychologique dont l'objet d'étude principal est le père; enfin, l'approche féministe qui met l'accent sur les relations de pouvoir entre les sexes, tant à l'intérieur de la famille que dans la sociétés.

2. Eva Thomas, Le viol du silence (Paris, Montaigne, 1986); Nathalie Schweighoffer, J'avais douze ans... (Paris, Fixot, 1986); Les enfants de l'inceste (Paris, Fixot, 1995); Shelley Sessions, L'amour inavouable. L'histoire vraie d'un inceste ordinaire (Paris, Presses de la Cité, 1991); Jean-Yves Soucy avec Annette, Cécile et Yvonne Dionne, Secrets de famille (Montréal, Libre Expression, 1995).

3. Pierre Scherrer, La sexualité criminelle en milieu rural (Paris, Masson, 1958), 72-87; L'inceste en milieu rural (Paris, Vrin, 1977); Jean-Claude Chesnais, Histoire de la violence en Occident de 1800 à nos jours (Paris, Laffont, 1981), 120; Michelle Rouyer et Marie Drouet, L'enfant violenté. Des mauvais traitements à l'inceste (Paris, Bayard, 1994), 183.

4. Robert Pauzé et Jacques Mercier, dir., Les agressions sexuelles à l'égard des enfants (Montréal, Éditions Saint-Martin, 1994), 34; Brigitte Camdessus et Michel C. Kiener, L'enfance violentée (Paris, ESF, 1993), 148; Shelley Sessions, op. cit., 16; Barbara Kavemann et Ingrid Lohstoter, Les pères criminels. La violence sexuelle contre les petites filles (Paris, Éditions des femmes, 1985), 127.

5. Les ouvrages sur l'inceste sont extrêmement nombreux. En plus des titres déjà cités, nous avons utilisé les suivants: Robert Pauzé et Jacques Mercier, dir., Les agressions sexuelles à l'égard des enfants (Montréal, Éditions Saint-Martin, 1994); Marceline Gabel, dir., Les enfants victimes d'abus sexuels (Paris, Presses Universitaires de France, 1992); Frédérique Gruyer, Martine Fadier-Nisse et Pierre Sabourin, La violence impensable. Inceste et maltraitance (Paris, 
Bien que les historiens aient l'habitude d'orienter leurs recherches vers des sujets qui intéressent leurs contemporains, peu d'entre eux ont essayé d'étudier l'inceste dans les sociétés passées, à cause de la rareté des sources. En France, Michel Kiener en a repéré quelques cas dans les archives judiciaires de la fin du $\mathrm{XIX}^{\mathrm{e}}$ siècle $^{6}$. On a surtout découvert que, dès cette époque, certains médecins avaient révélé que les abus sexuels contre les enfants, y compris l'inceste, existaient dans tous les milieux, même les plus «éclairés ${ }^{7}$ ». Aux États-Unis, la chercheure féministe Linda Gordon a étudié la question en se basant sur les archives des sociétés de protection de l'enfance à Boston, de 1880 à $1960^{8}$. Pour le Québec, Serge Gagnon interprète le silence habituel des confesseurs sur ce sujet et la réaction très vive de l'évêque quand l'inceste se produit comme une indication de la rareté extrême du phénomène'. Par contre, L'histoire des femmes au Québec cite le témoignage d'une prostituée qui affirme que l'inceste était chose courante à la campagne ${ }^{10}$.

Les archives judiciaires, si précieuses pour l'histoire de la famille, constituent également une source privilégiée pour étudier ce délicat problème. En effet, à partir de 1890 , l'inceste, c'est-à-dire les relations sexuelles entre «tout père ou mère et son enfant, tout frère et sœur, et tout aïeul ou aïeule et son petit-enfant», a été considéré comme un crime au Canada et puni très sévèrement: la sentence pouvait aller

Nathan, 1991); Alice Miller, L'enfant sous terreur. L'ignorance de l'adulte et son prix (Paris, Aubier, 1986); Leilla Sebbar, On tue les petites filles (Paris, Stock, 1978); Florence Rush, Le secret le mieux gardé, l'exploitation sexuelle des enfants (Paris, Denoël-Gonthier, 1983); J'ai commis l'inceste. Témoignages recueillis par Gilles David (Montréal, Éditions de l'Homme, 1995); Lise Julien et Isabelle Saint-Martin, L'inceste envers les filles: état de la situation (Québec, Conseil du statut de la femme, 1995); Susan Forward et Craig Buck, Betrayal of Innocence. Incest and its Devastation (New York, St. Martin's Press, 1978); Judith Lewis Herman et Lisa Hirschman, Father-Daughter Incest (Cambridge, Mass., Harvard University Press, 1981); Annie Imbens et Ineke Jonker, Christianity and Incest (Minneapolis, Fortress Press, 1992).

6. Brigitte Camdessus et Michel C. Kiener, op. cit., 53-54, 70-71.

7. En particulier les travaux de Tardieu et du docteur Bernard. Frédérique Gruyer et al., op. cit., 7-8; Michelle Rouyer et Marie Drouet, op. cit., 9-10; Jeffrey Moussaieff Masson, Le réel escamoté. Le renoncement de Freud à la théorie de la séduction (Paris, Aubier, 1984), 35-72.

8. Linda Gordon, «Incest and Resistance: Patterns of Father-Daughter Incest, 18801930», Social Problems, 33,4 (avril 1986): 253-267 et Heroes of their Own Lives. The Politics and History of Family Violence, Boston 1880-1960 (New York, Viking, 1988), chap. 7, «"Be Careful About Father", Incest, Girls' Resistance, and the Construction of Feminity». Voir aussi Lloyd Demause, «The Universality of Incest», The Journal of Psychohistory, 19,2 (automne 1991): 123-164; Brett Dahr, «The Sexual Molestation of Children: Historical Perspectives», The Journal of Psychohistory, 19,2 (automne 1991): 191-214.

9. Serge Gagnon, Plaisir d'amour et crainte de Dieu. Sexualité et confession au BasCanada (Sainte-Foy, Presses de l'Université Laval, 1990), 146-152.

10. Micheline Dumont et al., Histoire des femmes au Québec depuis quatre siècles (Montréal, Le Jour, 1992), 515. 
jusqu'à 14 ans de prison en plus de la peine du fouet pour l'homme ${ }^{11}$. La fille, considérée comme complice, risquait la même peine d'emprisonnement, à moins de prouver que la relation lui avait été imposée par la force. À partir de 1917, une peine de deux ans d'emprisonnement menaçait également «tout individu qui, étant beau-père ou bellemère, père ou mère nourricier ou tuteur, séduit ou a un commerce illicite avec son beau-fils ou sa belle-fille, son enfant adoptif ou pupille ${ }^{12} \gg$. Avant 1890 , une accusation de viol pouvait être portée contre les pères incestueux, tandis que les relations incestueuses librement consenties pouvaient faire l'objet de poursuites criminelles si elles revêtaient un "caractère notoire et public ${ }^{13}$ ».

Les cours de justice du Québec, chargées de juger les affaires criminelles, et le procureur général qui supervisait leurs activités eurent donc à traiter ce genre de crime plus d'une fois. Le dépouillement systématique des dossiers du Département du procureur général pour la période de 1867 à 1938 a permis de reconstituer 198 dossiers, auxquels nous avons ajouté quelques dizaines de procès repérés dans les archives des districts judiciaires de Québec, Montmagny, la Malbaie et la Beauce pour la période $1850-1938^{14}$ (tableau 1). Comme le premier de ces districts est urbain et les 3 autres ruraux, nous croyons que cet ajout n'entraîne pas de biais dans notre documentation. Nous atteignons ainsi un total de 217 cas correspondant à autant de familles soupçonnées d'inceste, et qui incluent 131 procès et 95 condamnations. Ces dossiers permettent d'étudier les aspects psychologique, familial et social de ce phénomène. Les déclarations faites par les membres de la famille et les voisins lors de l'enquête permettent de reconstituer la vie de la famille incestueuse et les réactions de l'entourage, tandis que les lettres échangées entre l'assistant du procureur général et ses subordonnés éclairent le fonctionnement du système judiciaire.

11. Code criminel du Canada (Ottawa, Imprimeur de la Reine, 1892), article 176, 81 . Cette loi est encore en vigueur, à l'exception de la peine du fouet, et ce, même s'il s'agit de relations entre adultes consentants.

12. Code criminel du Canada (Ottawa, T. Mulvey, 1920), art. $213 \mathrm{a}, 63$.

13. ANQQ, Dossiers du procureur général, 1880, $\mathrm{n}^{\circ} 3055$.

14. ANQQ, Dossiers du procureur général, 1867-1938. (Les limites chronologiques correspondent au début et à la fin de ce fonds d'archives.) Dossiers de la Cour des sessions de la paix, de la Cour du banc du roi et de la Cour d'appel de Québec, Montmagny, la Malbaie et la Beauce, des débuts à 1938. (Ces dossiers avaient été repérés lors d'une recherche antérieure portant sur l'histoire des filles-mères dans la région de Québec.) Nous avons repéré 40 cas d'inceste dans les dossiers de ces cours de justice, mais 21 d'entre eux figuraient déjà dans les dossiers du procureur général. Nous les avons donc comptés une seule fois. Pour des raisons de discrétion évidentes, nous ne citerons aucun nom de famille et n'indiquerons pas non plus les lieux de résidence. 


\section{Tableau 1}

Nombre de dossiers relatifs à des familles incestueuses ou soupçonnées d'inceste 1858-1938

\begin{tabular}{lccr}
\hline Décennie & $\begin{array}{c}\text { Dossiers du } \\
\text { procureur } \\
\text { général }\end{array}$ & $\begin{array}{c}\text { Cours de } \\
\text { justice }\end{array}$ & TOTAL \\
\hline $1850-1859$ & - & 1 & 1 \\
$1860-1869$ & - & 1 & 1 \\
$1870-1879$ & - & 1 & 0 \\
$1880-1889$ & 1 & 1 & 2 \\
$1890-1899$ & 10 & 1 & 5 \\
$1900-1909$ & 4 & 6 & 6 \\
$1910-1919$ & -2 & 4 & 43 \\
$1920-1929$ & 37 & 19 & 217 \\
$1930-1938$ & 144 & 148 \\
\hline TOTAL & 198 & & \\
\hline
\end{tabular}

Sources: ANQQ, Correspondance du procureur général, Cour des sessions de la paix et Cour du banc du roi de Québec, Montmagny, la Malbaie et la Beauce, Cour d'appel de Québec.

Cette documentation se prête principalement à une étude de cas, même si certaines statistiques élémentaires demeurent importantes. La comparaison de nos données avec celles de Linda Gordon et d'autres études effectuées dans différents pays au cours des vingt dernières années permettra de faire ressortir les traits universels de l'inceste, c'est-à-dire ceux que l'on observe au Québec avant 1940 et que l'on retrouve dans l'Europe et l'Amérique du Nord d'aujourd'hui. Nous tâcherons en même temps de souligner la spécificité de la situation québécoise. Le Québec traditionnel était une société patriarcale, où les lois civiles et religieuses reconnaissaient le père comme chef de famille. La coutume de la bénédiction paternelle montre bien quel prestige l'entourait, son autorité étant présentée comme venant de Dieu. Les enfants, eux, devaient respect et obéissance à leurs parents. Cette autorité, renforcée par le droit incontesté de corriger physiquement ses enfants «pour leur bien», ouvrait la porte à bien des abus ${ }^{15}$. Mais le sociologue Léon Gérin, qui a étudié la famille rurale québé-

15. D'après le titre du livre d'Alice Miller, C'est pour ton bien. Racines de la violence dans l'éducation de l'enfant (Paris, Aubier, 1984). 
coise au tournant du siècle, a souligné que l'autorité du père était tempérée par celles de la mère et du curé ${ }^{16}$. D'autre part, dans le domaine de la sexualité, l'Église catholique imposait des règles de conduite très restrictives (nullement inspirées par le respect de la personne, mais présentées comme la volonté de Dieu) et contrôlait les conduites par le biais de la confession ${ }^{17}$. Ainsi, les enfants confrontés au dilemme d'obéir à un parent qui leur demandait «d'offenser Dieu» pouvaient demander conseil à leur confesseur.

Dans quelle mesure la surveillance exercée par les mères et les curés a-t-elle pu contribuer à prévenir ou à réduire le nombre de cas d'inceste? Cela est impossible à savoir. Mais on peut essayer de voir comment les familles incestueuses s'efforçaient de concilier cette situation avec leur religion, et comment ce problème était traité par les autorités religieuses et l'appareil judiciaire. Notre attention se portera surtout sur les cas d'inceste entre père et fille, les plus nombreux, mais les autres ne seront pas oubliés.

\section{1 - LES LIMITES D'UNE SOURCE}

Pour porter un cas d'inceste à l'attention des tribunaux, la procédure la plus simple consistait à formuler une «dénonciation et plainte» devant un chef de police, un juge de paix ou un autre magistrat. Le juge émettait alors un mandat d'arrestation contre le présumé agresseur, le faisait emprisonner ou le laissait en liberté sous caution. Suivait une enquête préliminaire qui se terminait par un verdict de nonlieu ou un acte d'accusation renvoyant le prévenu subir son procès, lequel pouvait se dérouler devant un juge seul ou devant un jury, au choix de l'accusé.

Les frais de la dénonciation et de l'enquête préliminaire incombaient aux personnes intéressées. Lorsque celles-ci étaient très pauvres, le ministère public défrayait les coûts, mais la crainte d'avoir à débourser de l'argent a pu en retenir plus d'un. Il existait heureusement une alternative: écrire au bureau du procureur général pour dénoncer le cas d'inceste. C'était la démarche privilégiée par les personnes qui ne voulaient pas s'impliquer directement, surtout les curés. Un détective était alors envoyé pour effectuer une enquête discrète, s'assurer du bien-fondé de la plainte et recueillir suffisamment de preuves pour obtenir une condamnation lors d'un procès. En effet, dans les cas d'inceste comme dans ceux de viol, la déclaration de la

16. Léon Gérin, «L'habitant de Saint-Justin», Mémoires de la Société royale du Canada, $2^{\text {e }}$ série, tome 4 (mai 1898): 139-216.

17. Serge Gagnon, op. cit. 
victime devait être absolument corroborée, c'est-à-dire confirmée par un témoin ${ }^{18}$.

Le détective rédige ensuite un rapport détaillé à l'intention du procureur général. Dans une cinquantaine de cas, il juge inutile d'entreprendre un procès. Parfois, il croit que l'accusation est sans fondement, surtout quand le dénonciateur est affligé d'une mauvaise réputation tandis que la personne accusée jouit de l'estime générale. Plus souvent, il est convaincu de la réalité de l'inceste, mais personne n'accepte de venir témoigner en cour. Enfin, il est souvent bien difficile de distinguer les faits réels des commérages. Le cas de John L. est particulièrement éclairant. Lors d'une première enquête, en 1928, les membres de sa famille et plusieurs voisins se disent moralement certains qu'il est le père des trois enfants de sa fille. Mais comme personne ne les a pris sur le fait, le procureur juge inutile d'entreprendre un procès basé seulement sur des rumeurs. Cinq ans plus tard, après une deuxième enquête, Malvina $L$. passe aux aveux et son père en fait autant. Il est alors condamné à six mois de prison ${ }^{19}$.

Enfin, dans une dizaine de cas, l'inceste est découvert par hasard, à l'occasion d'une enquête sur un cas d'infanticide, d'enfant maltraité ou autre. En tout, nous avons repéré 119 dénonciations effectuées par lettre au procureur général et 59 faites directement à la police ou à un juge. L'état des dossiers ne permet pas de savoir comment les autres cas ont été connus. La personne qui prenait l'initiative de dévoiler l'affaire était parfois la victime elle-même ( $35 \mathrm{cas})$, seule ou avec quelqu'un d'autre, sa mère ( 25 cas) ou une autre parente (17 cas), un voisin ( 21 cas), et surtout le curé (51 cas) ou un autre ministre du culte $(4 \mathrm{cas})^{20}$. Ces derniers étaient informés soit par la victime, soit par sa famille ou simplement par la rumeur publique.

Les dossiers retrouvés dans la correspondance du procureur général sont très disparates. Les plus riches comptent des dizaines de pages et révèlent tous les détails de l'affaire, du début à la fin. D'autres se réduisent à une simple lettre portant sur des détails administratifs. Les données statistiques établies dans de telles conditions doivent donc être interprétées avec beaucoup de prudence.

Le fait le plus évident est l'augmentation des cas rapportés après 1920, et leur nombre serait encore plus élevé si les archives des cours

18. ANQQ, Dossiers du procureur général, 1938, $\mathrm{n}^{\circ} 13613$.

19. ANQQ, Dossiers du procureur général, 1928, $\mathrm{n}^{\circ} 1085$.

20. Parmi les autres dénonciateurs possibles, mentionnons le maire et les conseillers municipaux: 4 cas, un médecin: 2 cas, une société de protection des femmes et des enfants: 3 cas, le coroner: 2 cas, le député: 1 cas. Dans les autres cas, il est impossible de connaître l'identité du dénonciateur. 
de justice n'avaient pas été élaguées précisément à partir de cette date, privant les chercheurs de la majorité des procès pour inceste. Cette multiplication des dossiers reflète-t-elle une augmentation du nombre réel d'incestes? D'autres explications nous semblent plus vraisemblables; d'abord, la croissance exponentielle de toutes les archives judiciaires à partir de 1920; ensuite, les progrès de l'alphabétisation et une plus grande diffusion des journaux qui contribuent à mieux renseigner la population - c'est ainsi qu'une femme décide de porter plainte en 1935 après avoir appris, en lisant un journal, que c'était un crime punissable par les tribunaux ${ }^{21}$; enfin, une volonté manifeste des pouvoirs publics d'intervenir même au sein des familles pour protéger les enfants. Le célèbre procès des époux Gagnon, parents de la petite Aurore «l'enfant martyre» date précisément de $1920^{22}$.

Étant donné que près de $90 \%$ des cas repérés se situent entre 1920 et 1938 , notre étude portera principalement sur cette période, mais aucune différence majeure n'apparaît dans les dossiers d'avant 1920.

Les cas mentionnés dans la correspondance du procureur général proviennent de 56 comtés sur un total possible de 69 , et le nombre de cas à chaque endroit varie entre 1 et 10 . Il est donc impossible de dire que l'inceste était plus répandu dans une région que dans une autre. Mais était-il plus fréquent en région rurale ou urbaine? En nous basant sur 115 cas où le lieu de résidence de la famille est absolument certain, nous constatons que dans $60 \%$ des cas, l'inceste a lieu dans un habitat isolé, sur une ferme ou en plein bois (tableau 2).

Le milieu social révélé par l'occupation du chef de famille est généralement modeste, les cultivateurs et les journaliers étant les plus nombreux (tableau 3). Lorsque les curés et les détectives formulent des commentaires, ils décrivent le plus souvent ces gens comme pauvres ( $36 \mathrm{cas}$ ), parfois isolés ( 8 cas), peu intelligents ( $20 \mathrm{cas}$ ), ou affectés d'un problème d'alcool (12 cas). Dans 2 cas seulement, ils les dépeignent comme une famille modèle en apparence, le père jouissant d'une excellente réputation. Quant aux notables et aux professionnels, ils sont totalement absents de notre corpus documentaire.

Parmi ces 217 familles, plus du tiers présentent des incestes multiples: par exemple, le père avec plusieurs de ses filles $(55 \mathrm{cas})$, ou une fille avec plus d'un homme de sa famille. Nous arrivons ainsi à un total de 363 liaisons incestueuses qui recouvrent toutes les formes

21. ANQQ, Dossiers du procureur général, 1935, n 2924.

22. Peter Gossage, «La marâtre: Marie-Anne Houde and the Myth of the Wicked Stepmother in Quebec», The Canadian Historical Review, 76,4 (décembre 1995): 563-597. 


\section{Tableau 2}

\section{Lieu de résidence des familles incestueuses ou soupçonnées d'inceste}

Vivent dans les bois

10 cas

Habitat isolé

$\begin{array}{lr}\text { Agriculteurs } & 36 \\ \text { Colons } & 4 \\ \text { Vivent sur une terre } & 5 \\ \text { Vivent dans un rang } & 3 \\ \text { Vivent dans un canton } & 8 \\ \text { Vivent dans un township } & 3\end{array}$

Vivent dans une agglomération

$\begin{array}{ll}\text { de } 200 \text { à } 2900 \text { habitants } & 15 \text { cas } \\ \text { de } 3000 \text { à } 99000 \text { habitants } & 18 \text { cas } \\ \text { de } 100000 \text { habitants et plus } & 13 \text { cas }\end{array}$

Total des cas de résidence connus

115 cas

Sources: ANQQ, Correspondance du procureur général; Hormidas Magnan, Dictionnaire historique des municipalités, 1925; Recensements du Canada, 1921 et 1931, reproduits dans Annuaire du Québec, 1938.

\section{Tableau 3}

\section{Milieu socioprofessionnel des familles incestueuses ou soupçonnées d'inceste}

Métiers de la terre et de la forêt

Agriculteurs, colons et autres ${ }^{1}$

Bûcherons

Chasseurs

Métiers divers

Cordonnier, soudeur, mécanicien, électricien, menuisier, chauffeur, pilote, mineur, pêcheur, barbier, gardien de nuit

Journaliers

Vagabonds et mendiants

Total des métiers connus 101

1. Nous incluons dans cette catégorie les familles qui vivent sur une terre, dans un rang, un canton ou un township.

Sources: voir le tableau 1. 


\section{Tableau 4}

Répartition des différents types d'inceste

\begin{tabular}{lr}
\hline Père et fille & 252 \\
Beau-père et belle-fille & 12 \\
Père adoptif et fille & 1 \\
Amant de la mère et fille & 1 \\
Frère et sœur & 49 \\
Demi-frère et demi-sœur & 8 \\
Grand-père et petite-fille & 10 \\
Oncle et nièce & 11 \\
Père et fils & 5 \\
Beau-père et beau-fils & 2 \\
Mère et fils & 1 \\
Indéterminé & 11 \\
\hline Nombre total de liaisons incestueuses & 363 \\
Nombre total de familles incestueuses & \\
(ou soupçonnées d'inceste) & 217 \\
\hline
\end{tabular}

Sources: voir le tableau 1.

possibles d'inceste (tableau 4). (Nous employons le terme «liaisons» parce qu'il s'agit habituellement de relations sexuelles répétées.)

Ces liaisons sont presque toujours de nature hétérosexuelle, une personne de sexe masculin prenant l'initiative. Dans 3 familles seulement, elles se déroulent entre des garçons et leur père ou beau-père. Les liaisons entre une fille et son père biologique sont de beaucoup les plus fréquentes: 69,2\%. Dans un seul cas, le soupçon d'inceste se porte contre une mère et son fils. Cela correspond à la situation actuelle décrite par les travailleurs sociaux: à l'intérieur du cadre familial, les filles sont victimes d'abus sexuels plus souvent que les garçons $^{23}$.

L'âge de la fille au début de la liaison se situe le plus souvent entre 11 et 15 ans, avec une prédominance à 13 ans, donc au début de la puberté (tableau 5). La pédophilie existe aussi, mais ce n'est pas la forme la plus fréquente d'inceste.

23. Robert Pauzé et Jacques Mercier, dir., Les agressions sexuelles à l'égard des enfants (Montréal, Éditions Saint-Martin, 1994), 18. 


\section{Tableau 5}

Âge des filles au début et à la fin de la liaison incestueuse d'après les déclarations

\begin{tabular}{ccc}
\hline Age de la fille & $\begin{array}{c}\text { Début de la liaison } \\
\text { (nombre de cas) }\end{array}$ & $\begin{array}{c}\text { Fin de la liaison } \\
\text { (nombre de cas) }\end{array}$ \\
\hline 5 & 4 & 0 \\
6 & 0 & 0 \\
7 & 5 & 1 \\
8 & 8 & 0 \\
9 & 7 & 4 \\
10 & 8 & 2 \\
11 & 12 & 10 \\
12 & 19 & 5 \\
13 & 34 & 11 \\
14 & 18 & 13 \\
15 & 22 & 17 \\
16 & 7 & 20 \\
17 & 6 & 19 \\
18 & 5 & 12 \\
19 & 2 & 6 \\
20 & 2 & 7 \\
21 & 0 & 2 \\
22 & 0 & 2 \\
23 & 1 & 0 \\
24 & 0 & 2 \\
30 & 0 & 1 \\
\hline Total & 160 & 134 \\
\hline
\end{tabular}

Sources: voir le tableau 1.

La durée des liaisons est connue dans 121 cas. La majorité d'entre elles durent 2 ans ou moins ${ }^{24}$. Elles se terminent le plus souvent vers 16 ans, âge où les filles prennent conscience de leurs droits et peuvent se rebeller contre des privautés non désirées. «À 13 ans, on connaît rien. À 19 ans, on connaît», dit justement l'une d'elles ${ }^{25}$. Exceptionnellement, certaines liaisons pouvaient durer une dizaine d'années, le père et la fille menant une véritable vie de couple.

24. La durée des liaisons se répartit ainsi: moins d'un an: 19 cas; 1 an: 19 cas; 2 ans: 29 cas; 3 ans: 14 cas; 4 ans: 9 cas; 5 ans: 12 cas; 6 ans: 6 cas; 7 ans: 6 cas; 8 ans: 3 cas; 9 ans: 2 cas; 13 ans: 2 cas.

25. ANQQ, Cour d'appel, 1938, n' 16622. 
L'âge de l'homme, indiqué dans 43 cas seulement, se répartit entre 14 et 69 ans $^{26}$. Lorsqu'il est de moins de 30 ans, cela correspond à une liaison entre frère et sœur ou oncle et nièce. Dans un seul cas, l'agression sexuelle est commise par des adolescents sur une enfant qui est presque un bébé: leur demi-sœur âgée de 21 mois $^{27}$. Ce geste, peut-être inspiré par un désir de vengeance contre une marâtre, demeure lui aussi exceptionnel.

Lorsque l'enfant est très jeune, l'homme se contente parfois d'attouchements ( 7 cas) et de baisers génitaux ( 2 cas), de la masturbation (celle du père: 10 cas), du coït inter-fémoral (10 cas) ou de la fellation (4 cas), considérée par les contemporains comme la pire des dépravations $^{28}$. Mais la plupart du temps, la pénétration est complète et, malgré les précautions prises pour éviter une grossesse, généralement au moyen du coït interrompu (9 mentions), 64 jeunes filles deviennent enceintes. L'issue de cette grossesse demeure parfois inconnue (6 cas), une dizaine disent avoir eu recours à des manœuvres abortives, 42 mettent leur enfant au monde ( 9 d'entre elles en ont même plus d'un) et, pour 6 autres, l'issue tragique est un infanticide, parfois répété.

\section{2 - VIVRE L'INCESTE: UNE HISTOIRE À TROIS OU PLUS ${ }^{29}$}

Comment l'inceste était-il vécu dans la famille traditionnelle québécoise, patriarcale et catholique? Il est possible de le savoir en nous basant sur 140 dossiers particulièrement bien documentés.

Une cinquantaine de pères incestueux sont veufs ou séparés de leur femme, ce qui les prive de leur partenaire sexuelle habituelle, mais leur confère en même temps une plus grande liberté d'action. Une fille, généralement l'aînée, est alors amenée à remplacer l'épouse. Aussitôt après l'enterrement de sa femme, Joseph B. annonce à la famille que Cécilia sera désormais responsable des travaux du ménage et du soin des enfants. Un mois plus tard, il l'oblige à partager le lit conjugal $^{30}$. Ce comportement, que Linda Gordon range sous la rubrique «inceste domestique» est fréquemment observé dans la région de

26. L'âge des hommes se répartit comme suit: 14 à 19 ans: 6 cas; 20 à 29 ans: 8 cas; 30 à 39 ans: 7 cas; 40 à 49 ans: 10 cas; 50 à 59 ans: 9 cas; 60 à 69 ans: 3 cas.

27. ANQQ, Dossiers du procureur général, 1935, $\mathrm{n}^{\circ} 3597$.

28. Il va sans dire que les enfants et même les adultes n'emploient pas ces termes techniques. Ils s'expriment dans un langage beaucoup plus familier.

29. D'après le titre du livre de Michelle Marois, Camille Messier et Louise Perreault, L'inceste: une histoire à trois et plus (Québec, Ministère de la Justice, 1982).

30. ANQQ, Dossiers du procureur général, 1933, nº 6070. 
Boston à la même époque ${ }^{31}$. «Il faut que tu remplaces ta mère»: cette objurgation peut même s'adresser à une fillette de $7 \mathrm{ans}^{32}$. La fille de John L. s'identifie si bien à ce rôle qu'elle cohabite avec son père pendant au moins 15 ans après le décès de sa mère. Lorsque sa sœur aînée lui propose de partir, elle répond: «Qu'est-ce que papa ferait sans moi $^{33}$ ?»

Mais la majorité des pères incestueux ont une femme bien vivante à leurs côtés. Parfois, la relation sexuelle avec la fille s'explique par un désir de vengeance de l'homme qui veut punir sa femme de l'avoir contrarié $^{34}$, et surtout d'avoir refusé de le «servir», euphémisme servant à désigner les relations sexuelles ${ }^{35}$. Certains hommes estiment que leur épouse doit être sexuellement disponible à tout moment, et que son absence (ou son refus?) les autorise à satisfaire leurs désirs avec quelqu'un d'autre. "Fallait rester à la maison pour faire ton devoir», déclare Alfred R. à sa femme qui a été hospitalisée pour une fausse-couche après 17 accouchements ${ }^{36}$.

La présence et la soumission sexuelle de la femme ne suffisent pas toujours à contenter le mari. Madame Henri G. rapporte les propos du sien: «Moi, j'étais pas bonne. J'étais comme une baratte. La petite fille, elle, était bonne. Elle n'était pas capable de passer qu'il venait en l'air tout de suite ${ }^{37}$.» François F. vit également avec sa femme, mais il ne veut pas d'elle, explique le détective, préférant se servir de ses filles et de ses petites-filles ${ }^{38}$.

Certains hommes se conduisent comme s'ils avaient le droit de disposer à leur guise des femmes de la famille. «Cette fille est à moi et $\mathrm{j}$ 'ai le droit d'en faire ce que je veux», affirment cinq pères de famille ${ }^{39}$, propos identiques à ceux de certains maris qui battent leur épouse $^{40}$. Quant à Jean M., qui a des relations sexuelles avec sa sœur

31. Linda Gordon, loc. cit., 253. Voir aussi Leïla Sebbar, op. cit., 173; Barbara Kavemann, op. cit., 25; Michelle Rouyer, op. cit., 192-199.

32. ANQQ, Dossiers du procureur général, 1927, nº 1263.

33. ANQQ, Dossiers du procureur général, 1928, $\mathrm{n}^{\circ} 1085$.

34. ANQQ, Dossiers du procureur général, 1932, n 3434.

35. La même expression est employée dans le roman Les filles de Caleb (Montréal, Québec-Amérique, 1985). L'auteure, Arlette Cousture, décrit un mari en train de «servir» sa femme et un étalon disposé à «servir» une pouliche. Les réalités sexuelles sont exposées en termes crus quoique non méprisants.

36. ANQQ, Dossiers du procureur général, 1935, n 3552. Voir aussi Frédérique Gruyer, op. cit., 110; et Barbara Kavemann, op. cit., 128-129. La même situation est décrite dans le roman de Alice Walker, La couleur pourpre (Paris, Laffont, 1984), 253 p.

37. ANQQ, Dossiers du procureur général, 1927, n 4613.

38. ANQQ, Dossiers du procureur général, 1920, $\mathrm{n}^{\circ} 4691$; et $1880, \mathrm{n}^{\circ} 3055$.

39. ANQQ, Dossiers du procureur général, 1935, $\mathrm{n}^{\circ} 3552 ; 1929, \mathrm{n}^{\circ} 2454 ; 1936, \mathrm{n}^{\circ} 1153$

40. Voir notre article «Les séparations de corps dans le district judiciaire de Montréal, 1795-1880», Revue d'histoire de l'Amérique française, 49,1 (été 95): 3-33. 
mariée, il déclare à son beau-frère qui proteste: «Lorsque je serai marié, tu feras la même chose avec ma femme ${ }^{41}$.»

Cette attitude de propriétaire découlant du système patriarcal ${ }^{42}$ côtoie un parfait mépris des femmes, considérées comme des objets sexuels dont le destin inéluctable est de servir un homme. «Il faut que tu y passes» et «Ce n'est pas pire avec moi qu'avec un autre», disent certains pères. C'est sans doute Louis Y., 62 ans, qui pousse le mépris le plus loin. Il justifie ses relations sexuelles avec sa petite-fille de 16 ans en disant: "Quand un chien a un os devant lui, il le ronge ${ }^{43}$.»

Comment ces hommes s'y prenaient-ils pour parvenir à leurs fins? On peut supposer que les enfants étaient incapables de résister à l'autorité de leur père, confondant peut-être attouchements sexuels superficiels et manifestations d'affection. Mais les adolescentes avaient appris dans leur petit catéchisme que «l'œuvre de chair» est interdite en dehors du mariage, même si l'inceste n'était pas mentionné explicitement. Quelques hommes affirment donc à leur fille que ce n'est pas péché avec leur père $(6 \mathrm{cas})$, preuve qu'ils connaissent tout de même les règles morales, même s'ils les enfreignent. $\mathrm{Si}$ François $\mathrm{F}$. a réussi à déflorer toutes ses filles, c'est en les maintenant dans une ignorance absolue, estime l'enquêteur ${ }^{44}$. D'autres prennent comme prétexte que c'est leur devoir de faire l'éducation sexuelle de leur enfant.

Quelques pères s'efforcent de se gagner l'assentiment de leur fille par des promesses de cadeaux (14 cas), des manifestations d'affection, ou en lui affirmant qu'elle est leur préférée ${ }^{45}$. Dans 3 cas, la fille semble avoir été une partenaire consentante et l'une affirme y avoir pris du plaisir ${ }^{46}$. Mais les menaces sont bien plus fréquentes: celle de les priver du nécessaire (6 cas), de les faire enfermer au couvent ( 3 cas) ou de les mettre à la porte $(5$ cas $)$. Quand ces moyens ne suffisent pas, bien des pères ont recours aux coups (28 cas), aux menaces de coups ( 17 cas) ou de mort ( 32 cas). Les mêmes moyens servent à les contraindre au silence après le fait.

Parfois, lorsque le père s'abstient de la contraindre par la force, la fille réussit à le repousser. "Avec Corinne, je l'ai essayée, mais pas d'affaire, elle n'a pas voulu», admet Thomas D. ${ }^{47}$ Mais la principale

41. ANQQ, Dossiers du procureur général, 1938, nº 8152.

42. Barbara Kavemann, op. cit., 23, 126, 131; Gilles David, op. cit., 16.

43. ANQQ, Dossiers du procureur général, 1936, $\mathrm{n}^{\circ} 8757$.

44. ANQQ, Dossiers du procureur général, 1920, $\mathrm{n}^{\circ} 4691$.

45. ANQQ, Dossiers du procureur général, 1934, $\mathrm{n}^{\circ} 7979 ; 1928, \mathrm{n}^{\circ} 746$.

46. ANQQ, Dossiers du procureur général, 1919, $\mathrm{n}^{\circ} 1978$.

47. ANQQ, Dossiers du procureur général, 1933, $\mathrm{n}^{\circ} 3362$. 
impression qui se dégage des déclarations des victimes est celle d'un profond sentiment d'impuissance - sentiment inspiré dès l'enfance par les corrections corporelles ( «Il a commencé jeune à me faire marcher de peur $\left.{ }^{48} \gg\right)$ et qui se poursuit lors des relations sexuelles imposées brutalement: «Ça faisait mal, je pleurais, il me tenait», liton dans maintes déclarations ${ }^{49}$.

Le sentiment de peur ou d'impuissance suscitait parfois une grande passivité chez la jeune fille. Un exemple extrême est fourni par Harriet P. Au cours d'une liaison incestueuse commencée par un viol à l'âge de 14 ans et qui dure 6 années, son père tue les 3 enfants qu'elle met au monde. Le premier est assommé contre le mur d'une grange; le second est enterré vivant dans la neige: à la saison du dégel, elle voit le petit cadavre tous les jours; le troisième est déposé dans la cave d'où elle l'entend crier pendant 3 jours. Ce n'est qu'à l'âge de 20 ans qu'elle quitte la ferme où elle vivait avec son frère aîné et son père. Le procureur attribue son long silence aux menaces que lui faisait probablement ce dernier ${ }^{50}$. Chose certaine, elle n'était pas plus capable de défendre sa progéniture que de se défendre elle-même.

Comment les épouses réagissaient-elles? Étaient-elles toutes complices et passives, comme on les a souvent dépeintes ${ }^{51}$ ? Dans certains cas, elles n'étaient au courant de rien, les filles n'osant rien dire, à cause des menaces proférées par leur père ou parce qu'elles craignaient la réaction de leur mère. Mais d'autres se décident à rompre le silence.

Une seule femme semble consentir sans trop de réticences à «prêter» sa fille à son concubin ${ }^{52}$, situation plus fréquente aujourd'hui ${ }^{53}$, mais exceptionnelle à l'époque. Trois autres refusent obstinément de prendre au sérieux les plaintes de leur fille. Mais plusieurs adressent des reproches indignés à leur mari, quitte à être battues par ce dernier qui les accuse d'être jalouses ${ }^{54}$. Une cinquantaine d'entre elles dénoncent leur mari ${ }^{55}$. Certaines le font aussitôt qu'elles sont mises au cou-

48. ANQQ, Dossiers du procureur général, 1932, n $\mathrm{n}^{\circ} 5739$.

49. ANQQ, Dossiers du procureur général, $1935, \mathrm{n}^{\circ} 657$. Alice Miller, op. cit., a très bien décrit ce sentiment d'impuissance, cette perte de dignité qui envahit les enfants battus.

50. ANQQ, Dossiers du procureur général, 1932, n 3502 . Voir Frédérique Gruyer, op. cit., 20.

51. Barbara Kavemann et Ingrid Lohstoter, op. cit., 115-124; Robert Pauzé et Jacques Mercier, op. cit., 21; Michelle Rouyer et Marie Drouet, op. cit., 208; Leïla Sebbar, op. cit., 171; Frédérique Gruyer, op. cit., 21-22, 123-130; Susan Forward et Craig Buck, op. cit., 44-54.

52. ANQQ, Cour des sessions de la paix de Québec, Enquêtes préliminaires, 1904.

53. Leîla Sebbar, op. cit., 210, 227.

54. ANQQ, Dossiers du procureur général, 1927, no 4613.

55. Certaines femmes écrivent au procureur général, d'autres portent plainte à la police, d'autres, enfin, se confient au curé qui écrit au procureur général. 
rant de la situation (environ 26 cas); d'autres, plus tardivement ( 25 cas). Madame Henri G., dont il a été question plus haut, a fermé les yeux pendant une dizaine d'années, mais elle trouve que son mari exagère quand il bat sa fille, Elmina, pour la contraindre à la fellation en présence des autres enfants ${ }^{56}$. Une trentaine d'autres font preuve d'une passivité forcée. Madame $\mathrm{S}$. conseille à sa fille de se plier aux exigences de son père plutôt que de se faire rouer de coups ${ }^{57}$. Dans 36 cas, le père a d'ailleurs la réputation d'être un tyran qui terrorise toute la famille et fait même peur aux voisins (13 cas).

Mais la dépendance économique à l'égard du mari pourvoyeur constitue sans doute la contrainte la plus forte. Ainsi, madame Antoine B. a surpris son mari en train de violer sa fillette de 11 ans, mais elle ne l'a pas dénoncé, même après la mort de l'enfant, et elle refuse de porter plainte. Au détective estomaqué, elle déclare qu'elle a besoin de son mari pour faire vivre ses 7 autres enfants. La fille ânée, qui a déjà eu un enfant de son père, est du même avis: elle ne veut pas que ce dernier aille en prison, car elle est trop pauvre pour aider sa mère ${ }^{58}$. Placées dans une telle situation, certaines femmes font de nécessité vertu et couvrent leur passivité du manteau de la résignation chrétienne. Lors du procès de son mari, madame Henri G. explique au procureur pourquoi elle a supporté si longtemps les viols et autres brutalités infligés à sa fille infirme:

R. Je n'avais pas encore eu ma première qu'il a commencé à me battre. Depuis ce temps que ça toujours été mal comme ça. [...] J'allais voir le curé. [II] disait qu'il allait prier pour moi. Je crois bien que c'est la bonne sainte Anne qui me conservait. Je me recommandais au Bon Dieu et à la bonne sainte Anne et le bon Dieu m'a conservée jusqu'à aujourd'hui.

Q. Vous ne trouviez pas cela [l'inceste] abominable?

R. Oui certain que je trouvais ça abominable, mais j'endurais ça aussi pour l'amour du bon Dieu ${ }^{59}$.

Des études récentes ont démontré que les femmes battues par leur mari développent un sentiment d'impuissance qui les rend incapables de se défendre elles-mêmes ou de défendre leur fille contre un con-

56. ANQQ, Dossiers du procureur général, 1927, $\mathrm{n}^{\circ} 4613$.

57. ANQQ, Dossiers du procureur général, 1929, $\mathrm{n}^{\circ} 2454$.

58. ANQQ, Dossiers du procureur général, 1931, n 5424 .

59. ANQQ, Correspondance du procureur général, 1927, $\mathrm{n}^{\circ} 4613$. Le livre déjà cité de Shelley Sessions montre comment une femme américaine cherche un réconfort dans le fondamentalisme religieux après avoir découvert la liaison entre son mari et sa fille. Mais la fortune du mari contribue également à la retenir. 
joint abusif ${ }^{60}$. Nous retrouvons cette situation explicitement dans une trentaine de familles incestueuses. À cela s'ajoute la dépendance économique des femmes qui était certainement plus grande à l'époque. Ce cercle vicieux d'impuissance et de dépendance qui se perpétue de mère en fille est nettement exprimé par un père qui répond aux gémissements de sa fille qu'il viole: «Endure, ça t'habituera pour plus $\operatorname{tard}^{61} . »$ Les choses n'étaient guère différentes dans les autres types de liaisons sexuelles: avec un oncle, un grand-père ou un frère, ce dernier cas étant le plus fréquent après les relations père-fille. Sans doute, les frères ne disposaient pas d'une autorité égale à celle des pères, mais ils n'hésitaient pas plus qu'eux à se servir de leur force ${ }^{62}$ et ils faisaient preuve de la même désinvolture, de la même attitude de propriétaire à l'égard du corps de leur sœur.

Le cas de la famille $F$. est particulièrement révélateur. Le frère aîné raconte comment, à l'âge de 17 ans, il eut sa première relation sexuelle avec sa sœur de 12 ans. Un jour qu'elle passait près de lui, sans dire un mot, il la jeta sur le lit et la viola, non sans difficulté la première fois. Quelques mois plus tard, le frère cadet, âgé de 16 ans, ayant appris la chose, décida de faire de même. Puis le plus jeune fut invité à suivre l'exemple de ses aînés ${ }^{63}$. Apparemment, après le premier viol, l'adolescente accepta les autres relations comme un fait inévitable.

Ajoutons que la contribution des garçons au budget familial incite parfois les parents à fermer les yeux sur leurs agissements. Quand les deux sœurs d'Ulysse G. demandent la protection de la police parce que leur frère les a violées et battues, leur père lui-même essaie de les convaincre de retirer leur plainte. "Ça pas de bon sens qu'il reste enfermé. C'est lui qui me donne de l'argent pour vivre. Vous autres, vous me donnez rien ${ }^{64} . »$ Cette dépendance économique, presque aussi grande que celle des mères de famille nombreuse à l'égard de leur mari, renforce le pouvoir des membres masculins de la famille.

Le comportement des pères incestueux, observé à travers les dossiers judiciaires québécois, correspond au portrait de tyran familial tel que dépeint par les chercheuses féministes ${ }^{65}$. Mais peut-on expliquer

60. Frédérique Gruyer et al., op. cit., 123-129, 164.

61. ANQQ, Dossiers du procureur général, 1938, n 2968. Voir Brigitte Camdessus, op. cit., 73-74.

62. ANQQ, Dossiers du procureur général, 1934, nº 3928.

63. ANQQ, Dossiers du procureur général, 1935, nº 333.

64. ANQQ, Dossiers du procureur général, 1934, n 7437.

65. En particulier Barbara Kavemann et Ingrid Lohstoter, Leïla Sebbar, et Annie Imbens et Ineke Jonker. Voir aussi Michelle Rouyer et Marie Drouet, op. cit., 184. 
ce comportement par des causes psychologiques? De nombreuses études ont démontré que les personnes ayant souffert d'abus sexuels dans leur enfance risquaient de devenir à leur tour des abuseurs ${ }^{66}$. Nos dossiers révèlent une seule confidence de ce genre: Joseph T. qui se fait masturber par sa fillette de 10 ans en lui disant que «ce n'est pas mal, d'autres se faisaient faire la même chose par lui lorsqu'il était jeune ${ }^{67}{ }_{\gg}$. Combien d'autres pères incestueux ont été des victimes dans leur enfance? Nos sources sont muettes à ce sujet, mais elles nous font connaître des familles où l'inceste se reproduit sur 2 générations, soit parce que l'homme s'en prend à ses filles et ensuite à ses petites-filles ( 3 cas) ou parce que le père incestueux engendre des fils qui à leur tour commettent l'inceste avec leurs filles (4 cas). Si l'on ajoute les 11 familles où la fille a des relations sexuelles avec son père et ses frères, force est de conclure que l'inceste est parfois un comportement appris dans la famille ${ }^{68}$.

\section{3 - FUIR L'INCESTE}

En dépit de ces contraintes physiques, psychologiques et économiques, certains cas d'inceste finissaient par être découverts. Parfois des rumeurs circulaient dans la paroisse, surtout quand se déclarait une grossesse hors mariage. Malgré les efforts de leur père pour les isoler du reste de la communauté, plusieurs jeunes filles font appel à l'aide de la parenté et des voisins. Ainsi, Marie et Cécile T. vont se réfugier chez un cousin pour échapper aux assauts de leur père ${ }^{69}$, tandis que Alice G. va montrer à une voisine les «preuves» des agissements du $\operatorname{sien}^{70}$.

Les curés jouent alors un rôle particulièrement important. Certaines jeunes filles lui confessent ce "péché» qui les préoccupe beaucoup $^{71}$. Ils peuvent aussi être informés par une autre source. Dans les cas que nous avons étudiés, ils prennent ces dénonciations au sérieux (à 2 exceptions près), écrivent au procureur général (51 cas) ou recourent à un autre moyen pour aider la victime (30 cas). Lorsque l'affaire n'a pas transpiré à l'extérieur, ils préfèrent souvent la régler discrètement. Il arrive qu'une verte semonce adressée au père suffise à chan-

66. Brigitte Camdessus, op. cit., 19; Frédérique Gruyer, op. cit., 40, 119; Michelle Rouyer, op. cit., 14, 210, 226; Marceline Gabel, dir., op. cit., 148; Gilles David, op. cit.

67. ANQQ, Dossiers du procureur général, 1937, $\mathrm{n}^{\circ} 6983$.

68. Frédérique Gruyer, op. cit., 102, 109, 177, 197-198; Brigitte Camdessus et Michel C. Kiener, op. cit., 103, 162; Michelle Rouyer, op. cit., 218.

69. ANQQ, Dossiers du procureur général, 1891, no 8531.

70. ANQQ, Dossiers du procureur général, 1938, n 12392.

71. Idem. 
ger son comportement ${ }^{72}$, mais, habituellement, il vaut mieux aider la fille à quitter le toit paternel.

Un emploi comme servante à l'extérieur du foyer est la solution pour 18 jeunes filles. Sept autres vont demeurer chez un membre de la parenté. L'hôpital de la Miséricorde peut offrir un refuge en cas de grossesse ( 5 cas). Les maisons du Bon-Pasteur et d'autres pensionnats accueillent une quinzaine de filles. Quatre d'entre elles décident d'y rester définitivement parce qu'elles ont trouvé un refuge sûr.

Plusieurs chercheurs ont souligné le fait que l'inceste mène à la prostitution $^{73}$. Certains y voient une conséquence de la perte de tout sentiment de dignité chez ces filles traitées depuis l'enfance comme des objets sexuels. D'autres considèrent ce mode de vie comme un geste d'autonomie et de révolte. Parmi les cinq cas de ce genre que nous avons relevés, une jeune fille exprime très bien cette ambiguiité quand elle déclare qu'elle aime mieux «faire la vie» avec des étrangers qu'avec son père ${ }^{74}$.

Le mariage constitue une autre porte de sortie, mais pas toujours facile, car certains pères incestueux se montrent extrêmement possessifs, interdisant à leurs filles de sortir et de rencontrer des garçons. Néanmoins, 26 mariages sont mentionnés dans nos sources. Dans 4 cas, le père (ou le frère) veulent quand même poursuivre la liaison incestueuse, mais ils doivent alors affronter un autre homme. Quand George M. se permet de battre sa fille mariée, le mari de celle-ci intervient: «It is not fair, dit-il à son beau-père, she is your daughter but she is my wife and it is my duty to protect her ${ }^{75}$.» Cette jeune femme a donc trouvé un protecteur efficace.

Le départ d'une jeune fille ne règle pas toujours le problème en profondeur, car le père risque alors de s'en prendre à celles qui restent avec lui. William B. le déclare nettement en voyant partir sa fille aînée, amenée par une tante: «Je vais mettre l'autre [la cadette] à ma main $^{76} . \gg$ C'est d'ailleurs souvent pour protéger leurs sœurs plus jeunes que les filles se décident à dénoncer leur père ${ }^{77}$. Yvonne $C$., par exemple, âgée de 17 ans, a déjà été violée par son père. Elle constate

72. ANQQ, Dossiers du procureur général, 1938, no 13613.

73. Frédérique Gruyer et al., op. cit., 25-30; Leïla Sebbar, op. cit., 278-279; Robert Pauzé et Jacques Mercier, op. cit., 85-88; Barbara Kavemann, op. cit., 90, 94-96; Linda Gordon, op. cit., 240-248.

74. ANQQ, Dossiers du procureur général, 1936, n 1153.

75. ANQQ, Cour des sessions de la paix de Québec, 1864.

76. ANQQ, Dossiers du procureur général, 1921, $\mathrm{n}^{\circ} 5196$.

77. Michelle Rouyer, op. cit., 203; Frédérique Gruyer, op. cit., 20; Barbara Camdessus et Michel Kiener, op. cit., 103. 
qu'il fait la même chose avec sa sœur de 11 ans et ses petits frères, même si «il s'en sert autrement». Avec l'assentiment de sa mère, elle écrit alors au procureur général «pour protéger les plus jeunes ${ }^{78}$ ».

\section{4 - PUNIR L'INCESTE}

L'arrestation du père constitue une solution radicale, mais à laquelle les personnes intéressées ne recourent pas sans réticences. En effet, l'emprisonnement du père signifie la disparition du gagne-pain de la famille et bien des femmes reculent devant cette perspective. Le curé et les autorités municipales aussi, car ils savent que la famille tombera à la charge de la paroisse qui n'est pas toujours capable d'assumer un tel fardeau ou disposée à le faire ${ }^{79}$.

Maria B., une immigrante italienne habitant Montréal, opte pour une solution mitoyenne. Aussitôt qu'elle s'aperçoit que son mari abuse sexuellement de sa fillette de 9 ans, elle demande la séparation de corps et la garde de ses enfants. Elle explique au juge qu'elle ne veut pas entreprendre de poursuites criminelles, car elle désire que son mari continue à travailler pour pouvoir lui payer une pension permettant de placer ses enfants au couvent. Quant à elle, «je peux gagner ma vie avec mon travail», déclare-t-elle fièrement ${ }^{80}$. Mais on recourait rarement à la séparation de corps: seulement 2 cas connus sur 217 , auxquels s'ajoutent une dizaine de séparations de fait, motivées dans la moitié des cas par la brutalité du mari ${ }^{81}$.

Malgré ces inconvénients majeurs, l'intervention des pouvoirs publics était demandée, surtout quand l'affaire était déjà bien connue et que les autorités voulaient faire cesser un scandale ou se débarrasser d'un indésirable qui déshonorait la paroisse ${ }^{82}$.

Les preuves recueillies par les détectives ou les policiers ne sont pas toujours suffisantes aux yeux des juges et, dans neuf cas, l'enquête préliminaire se termine par un non-lieu. Dans une dizaine d'autres affaires, la jeune fille ou la mère retirent leur plainte, alléguant qu'elles veulent préserver l'honneur de la famille, qu'elles ont besoin du revenu gagné par le père, qu'il a promis de ne plus recommencer,

78. ANQQ, Dossiers du procureur général, 1932, n 5739.

79. ANQQ, Dossiers du procureur général, 1935, nº 6276.

80. ANQM, Cour supérieure, $1925, \mathrm{n}^{\circ} 670$.

81. D'autre part, l'analyse de 753 procès en séparation de corps dans le district judiciaire de Montréal, de 1795 à 1930, a permis de repérer seulement une dizaine de cas où l'inceste est mentionné. Voir notre article «Les procès en séparation de corps dans la région de Montréal, 1900-1930», à paraître.

82. ANQQ, Dossiers du procureur général, 1930, nº 5903. 
qu'elles lui ont pardonné, etc. Ce qu'elles désirent, au fond, ce n'est pas que l'abuseur soit mis en prison, mais simplement qu'il renonce à ses comportements abusifs. Le procureur général est habitué à voir des jeunes filles faire des aveux au détective, puis se rétracter une fois rendues en cour ${ }^{83}$. Dans les lettres qu'ils échangent avec lui, les curés et les détectives expriment leur frustration devant de telles situations, encore fréquentes aujourd'hui ${ }^{84}$.

Compte tenu du fonctionnement du système judiciaire, les accusés ont tout intérêt à nier les faits, surtout s'il n'y a pas de témoins, car le juge et le jury doivent faire jouer le bénéfice du doute en leur faveur. Néanmoins, 42 d'entre eux passent aux aveux tout en essayant d'atténuer la gravité de leurs actes en évoquant, par exemple, le consentement de la fille $(7 \mathrm{cas})$ ou le fait qu'elle était déjà déflorée ( 5 cas), arguments que l'on retrouve aussi dans les procès pour viol ou séduction.

Les spécialistes de différentes disciplines qui ont analysé le comportement des hommes incestueux sont frappés par l'absence de sentiment de culpabilité et la similitude des arguments qu'ils emploient pour se justifier dans tous les pays où de telles études ont été effectuées $^{85}$ : ou ils revendiquent le droit de disposer du corps de leur fille ou ils se posent en victimes d'une femme frigide, de la boisson, etc. Ces rationalisations sont également courantes au Québec avant 1940. Il est même surprenant de voir cinq hommes exprimer quelques regrets, aussitôt suivis d'une tentative de justification.

L'arrestation et la perspective de l'emprisonnement constituent néanmoins un choc pour certains de ces hommes: deux d'entre eux se suicident. Mais ce geste ne reflète pas pour autant un sentiment de culpabilité. Dans sa lettre d'adieu, John C. reproche à sa fille de l'avoir dénoncé, ajoutant qu'il lui pardonne «pour lui montrer qu'il a bon cœur ${ }^{86}{ }^{\prime}$.

Les juges qui s'occupent des affaires d'inceste sont visiblement partagés entre le désir de faire cesser une situation qui soulève la réprobation et le devoir de faire respecter les droits des accusés. Une décision mal fondée risque d'être renversée par la Cour d'appel: voilà un autre motif qui les incite à la prudence.

83. ANQQ, Dossiers du procureur général, 1928, nº 4799.

84. Frédérique Gruyer, op. cit., 182.

85. Frédérique Gruyer et al., op. cit., 108-114; Barbara Kavemann et Ingrid Lohstoter, op. cit., 128-136; Gilles David, op. cit.

86. ANQQ, Dossiers du procureur général, 1932, nº 2617. 
Le marchandage de sentence (plea bargaining) est une avenue souvent utilisée, surtout quand le procureur n'est pas absolument certain que les jurés seront convaincus «hors de tout doute raisonnable» de la culpabilité de l'accusé. Plutôt que de s'exposer à l'issue incertaine d'un procès devant jury, près d'une trentaine d'accusés optent pour un procès devant un juge seul et acceptent de plaider coupables (d'inceste ou d'un autre délit sexuel) afin de susciter la clémence de la Cour $^{87}$.

Lorsque l'accusé persiste à plaider non coupable, la crédibilité de la victime et de ses témoins revêt une importance cruciale. Comme dans les procès pour viol ou séduction, il faut que les jeunes filles aient une réputation absolument irréprochable pour qu'on se fie à leur témoignage. Si la victime admet avoir eu des relations sexuelles avec un autre homme, en plus de son père ${ }^{88}$, ou si un témoin a une réputation douteuse, comme Bernadette R. accusée de vagabondage ${ }^{89}$, elles ne sont pas crues et l'accusé est acquitté.

Malgré ces difficultés, la majorité des hommes qui subissent leur procès au-delà de l'enquête préliminaire sont reconnus coupables et condamnés à une peine de prison plus ou moins longue selon les circonstances (tableau 6). Dans la moitié des cas connus, la sentence ne dépasse pas deux ans. Les sentences les plus légères (moins de un an de prison) sont imposées soit parce que la preuve n'est pas suffisante pour un procès avec jury, soit parce que la relation sexuelle n'a pas été complète, ou encore parce que l'accusé semble décidé à ne pas récidiver et que sa famille demande sa libération. Dans les cas plus graves, et lorsque les preuves sont irréfutables, le juge peut se permettre de «faire un exemple», sans craindre de voir son verdict renversé par un tribunal supérieur. La sentence la plus lourde, l'emprisonnement à perpétuité, est imposée à Henry $\mathrm{P}$., non pour inceste mais pour manslaughter, parce qu'il a tué trois nouveau-nés, mais il est fort possible que le juge ait tenu compte de sa liaison incestueuse avec sa fille $^{90}$.

La peine du fouet n'est imposée qu'exceptionnellement, à 3 hommes dépeints comme des brutes et des ivrognes, qui ont battu

87. Les actes d'accusation se répartissent comme suit: inceste: 91 , tentative d'inceste: 4, viol: 6 , tentative de viol: 4 , séduction de mineure: 1 , commerce charnel avec une fille de moins de 14 ans: 6 , attentat à la pudeur: 11 , assaut indécent: 5 , menaces de mort: 1 , suppression de part: 2 , manslaughter: 1 ; total: 132 . Il est possible de porter deux accusations contre une même personne.

88. ANQQ, Dossiers du procureur général, 1922, $\mathrm{n}^{\circ} 1528$.

89. ANQQ, Dossiers du procureur général, 1929, $\mathrm{n}^{\circ} 119$.

90. ANQQ, Dossiers du procureur général, 1932, n 3502. 


\section{Tableau 6}

Répartition des sentences

\begin{tabular}{lr}
\hline Sentences inconnues & 11 \\
Sentences suspendues & 4 \\
11 jours à 11 mois de prison & 25 \\
12 à 23 mois de prison & 10 \\
24 à 30 mois de prison & 16 \\
3 ans de prison & 12 \\
4 ans de prison & 7 \\
5 ans de prison & 10 \\
7 ans de prison & 2 \\
8 ans de prison & 2 \\
10 ans de prison & 1 \\
12 ans de prison & 1 \\
24 ans de prison & 1 \\
28 ans de prison & 2 \\
Emprisonnement à perpétuité & 1 \\
10 ans de prison et fouet & 1 \\
7 ans de prison et fouet & 1 \\
4 ans de prison et fouet & 1 \\
2 ans à l'école de réforme & 1 \\
3 ans à l'école de réforme & 1 \\
Envoyés à l'asile d'aliénés & 3 \\
Acquittés & 18 \\
\hline Total des procès & 131 \\
\hline
\end{tabular}

Sources: voir le tableau 1.

et menacé de tuer leurs filles qui essayaient de leur résister ${ }^{91}$. L'histoire d'Alice et de Jeanette G. a tout pour émouvoir le juge le plus blasé. Un mois après la mort de sa femme, il viole ses 2 filles, âgées de 11 et 13 ans. Elles tentent en vain de lui échapper en se cachant sous le lit ou dans le grenier. Les relations continuent pendant 6 ans, même après son remariage. Elles en parlent à leur belle-mère qui répond que «ces choses-là peuvent pas arriver»; au curé qui refuse de les croire; à leur grand-mère, qui les fait battre par leur père. Finalement, une voisine les prend sur le fait, près de l'étable. Devant le tribunal, le père affirme que ses filles étaient consentantes et venaient

91. ANQQ, Dossiers du procureur général, 1935, n 3552; 1938, n 12392; Cour d'appel, 1929-1930, n० 1448 . 
même le chercher. Le juge le condamne alors à 7 ans de pénitencier et 6 coups de fouet ${ }^{92}$. Les sentences de 7 ans et plus s'expliquent toutes par des circonstances aggravantes: relations avec plus d'une fille, coups et blessures infligés à l'épouse et aux enfants. Dans 3 de ces cas, le procureur souligne que la communauté est indignée. La sévérité de la sentence a donc pour but de la satisfaire.

Qu'advient-il de l'accusé et de sa famille par la suite? John L. revient chez lui où il continue de cohabiter avec sa fille ${ }^{93}$. Ernest B., libéré faute de preuves, maltraite sa famille plus que jamais à son retour, à la fois pour se venger de son arrestation et pour montrer qu'il est maître chez lui ${ }^{94}$. Enfin, deux femmes demandent la libération de leur mari parce qu'elles ont plusieurs enfants en bas âge et qu'elles sont dans la misère sans lui. Elles se disent convaincues que le temps passé en prison a suffi à les guérir et qu'ils ne recommenceront plus $^{95} \ldots$

\section{CONCLUSION}

L'isolement de certaines familles en milieu rural, l'attitude menaçante du père, la crainte de perdre le gagne-pain de la famille, la réticence des filles à témoigner devant les tribunaux, voilà autant de facteurs qui contribuaient à faire de l'inceste un secret bien gardé dans la société traditionnelle québécoise.

Au terme de cette recherche, on ne peut qu'être frappé par la similitude des comportements observés au Québec avant 1938 de même qu'en Europe et en Amérique du Nord à l'époque actuelle. Le comportement des pères incestueux d'abord, sûrs de leur bon droit et dépourvus de culpabilité; celui des filles, soumises très tôt à une autorité qui s'exerce par la force et engendre chez elles un sentiment d'impuissance; celui des épouses dont la passivité s'explique surtout par la dépendance économique, peut-être plus grande en milieu rural, et certainement plus prononcée quand la famille compte de nombreux enfants. Tout cela s'inscrit très bien dans le cadre d'analyse féministe qui considère l'inceste et le viol comme des manifestations des relations de pouvoir entre hommes et femmes.

Dans quelle mesure la religion catholique influençait-elle le comportement des familles incestueuses? Certains hommes niaient que

92. ANQQ, Dossiers du procureur général, 1938, n 12392; Cour des sessions de la paix et Cour d'appel, $\mathrm{n}^{\circ} 16722$.

93. ANQQ, Dossiers du procureur général, 1928, n 1085.

94. ANQQ, Dossiers du procureur général, 1934, n ${ }^{\circ} 1638$.

95. ANQQ, Dossiers du procureur général, 1932, no 5739 et $1936, n^{\circ} 8554$. 
leur acte fût un péché. D'autres l'admettaient, mais cet aveu ne suffisait pas à modifier leur comportement. Hormidas $\mathrm{P}$. promet à sa nièce de ne plus la toucher, parce que «cela fait pleurer le petit Jésus», mais il recommençait le lendemain, dit-elle ${ }^{96}$.

De leur côté, certaines filles essaient de repousser leur père en lui disant que «c'est un péché». Le désir de bien pratiquer leur religion et la crainte de se voir refuser l'absolution les incitent à fuir la relation incestueuse. Comme Linda Gordon le faisait remarquer, l'appel à une autorité supérieure, celle de Dieu, permet à ces filles d'échapper aux abus de pouvoir de leur père ${ }^{97}$.

Chez les femmes mariées, la motivation est moins claire. Madame Henri G. déclare qu'elle endurait les violences de son mari contre elle et sa fille «pour l'amour du bon Dieu». Maria B., par contre, refuse absolument de laisser son mari commettre «des actes de bestialité» avec sa fille ou avec elle. «Je suis prête à faire ce que Jésus-Christ commande», dit-elle. La religion pouvait donc servir à justifier la résignation aussi bien que la révolte. Ces recours à l'autorité divine révèlent aussi la difficulté pour les femmes et les filles d'affirmer leurs droits en tant qu'être humain dans la famille et la société patriarcales québécoises. Nous sommes ici aux antipodes du patriarcat mitigé et bienveillant décrit par Léon Gérin au tournant du siècle, puis reconstitué par Denise Lemieux à partir des récits de vie et des autobiographies $^{98}$.

La société rurale traditionnelle offrait heureusement des moyens de recours. Dans les dossiers que nous avons étudiés, la parenté, les voisins et le curé répondent habituellement aux appels à l'aide. Les établissements des communautés religieuses, en particulier la Miséricorde et le Bon-Pasteur, offrent également un abri. Là aussi, un effet pervers est possible, car certains pères exploitent la crainte de leurs filles de «se faire enfermer». Mais celles qui ont connu l'inceste disent qu'elles préfèrent le couvent, du moins pour un temps. À une époque où l'on dénonce les agressions sexuelles commises dans les institutions religieuses pour garçons, il n'est que juste de rappeler le rôle positif joué par d'autres personnes religieuses dans ce domaine.

Les dossiers que nous avons reconstitués ne nous donnent qu'une vue bien incomplète de ce phénomène. À côté des 217 cas portés au

96. ANQQ, Dossiers du procureur général, 1936, nº 2870.

97. Linda Gordon, op. cit., 260.

98. Denise Lemieux et Lucie Mercier, Les femmes au tournant du siècle, 1880-1940. Âges de la vie, maternité et quotidien (Sainte-Foy, Institut québécois de recherche sur la culture, 1989). 
grand jour, combien sont restés dans l'ombre parce que les victimes n'osaient pas briser le mur du silence et de l'isolement? Des études récentes estiment que la fréquence de l'inceste se situe entre 15 et $20 \%$ dans la population en général ${ }^{99}$, et que la majorité $(81 \%)$ des victimes d'agression sexuelle pendant l'enfance n'ont jamais demandé d'aide ${ }^{100}$. Il n'est pas impossible que les chiffres soient aussi élevés pour le Québec d'avant 1938. Des recherches plus poussées dans les archives des maisons du Bon-Pasteur et celles des cours de justice permettront sans doute de révéler bien d'autres aspects de ces secrets de famille.

99. Hélène Hamel, dir., Survivre à l'inceste: mieux comprendre pour mieux intervenir (Cowansville, La collective par et pour elles, 1989), 175 et Comité canadien sur la violence faite aux femmes, Un nouvel horizon, éliminer la violence, atteindre l'égalité (Ottawa, Approvisionnements et Services Canada, 1993), cités dans Lise Julien et Isabelle Saint-Martin, L'inceste envers les filles: état de la situation (Québec, Conseil du statut de la femme, 1995), 22.

100. Rapport Badgley, Les infractions sexuelles à l'égard des enfants (Ottawa, Approvisionnements et Services Canada, 1984), cité dans Robert Pauzé et Jacques Mercier, op. cit., 14. 